\title{
WHI High-Speed Streams at Geospace
}

\author{
G. Maris ${ }^{1}$ and O. Maris ${ }^{2}$ \\ ${ }^{1}$ Institute of Geodynamics, RO-020032, Bucharest, Romania \\ email: gmaris@geodin.ro \\ ${ }^{2}$ Institute for Space Sciences, RO-077125, Bucharest, Romania \\ email: maris@spacescience.ro
}

\begin{abstract}
The fast solar wind analysis during the Whole Heliospheric Interval (Carrington Rotation no. 2068: March 20 - April 16, 2008) is herein presented. The analysis is based on the data recorded by space experiments aboard the ACE and SOHO missions. The high-speed streams in the solar wind were determined by their main parameters: duration, maximum velocity, velocity gradient. The fast solar wind was also analyzed during the preceding and following Carrington Rotations, nos. 2067 and 2069, respectively. The main properties of the rapid streams and their solar sources were analysed in terms of the present solar minimum phase. The geospace response to the fast streams was evaluated using the geomagnetic index dynamics. A comparative analysis of the high-speed streams registered during Whole Heliospheric Interval with the ones determined during Whole Sun Month was also made.
\end{abstract}

Keywords. solar wind - high-speed streams, solar-terrestrial relations

\section{Introduction}

An internationally coordinated observing and modeling program, Whole Heliospheric Interval (WHI), focused on observations originating from Carrington Rotation (CR) 2068, March 20 - April 16, 2008 was developed during IHY. The interval was chosen during the prolonged minimum between solar cycles 23 and 24, one solar cycle after Whole Sun Month (WSM), August 10-September 8, 1996, in order to make a comparative analysis of solar quiet intervals near solar cycle minima. The 3 -D observing views of the Sun during CR 2068 were possible aboard STEREO, SOHO, ACE missions in quadrature with Ulysses (operated at a reduced rate). Using all ground and space data, it would be possible to accomplish the main goals of the program: to characterize the 3 -D solar minimum heliosphere and to trace the effects of solar structure and activity through the solar wind to the Earth and other planetary systems. This paper presents a brief overview of the high-speed streams (HSSs) in the solar wind that appeared during WHI as well as during the two adjacent Carrington Rotations, 2067 and 2069. A comparison of the HSSs registered during WHI with the ones during WSM is made. The HSS impact on the terrestrial magnetosphere, evaluated by Ap and Dst geomagnetic index dynamics and by a regression line analysis, ends the paper.

\section{HSSs during WHI}

The HSSs have a multitude of definitions given by different authors; the simplest is often the best: a large increase in the solar wind velocity lasting for several days. Some catalogues of HSSs were set up (Lindblad and Lundstedt, 1989 and the references therein: Mavromichalaki et al. 1998; Maris and Maris, 2008) covering solar cycles 2023. We extended the HSS catalog to 2008, during WHI. We used the same selection procedure of the streams as Linblad and Lundsted because it allows for a more precise 
determination of the HSS beginning and end. So, we selected as a 'high-speed stream' a solar wind flow having $\triangle \mathrm{V} 1 \geqslant 100 \mathrm{~km} / \mathrm{s}$ that lasted for two days, where $\triangle \mathrm{V} 1$ was the difference between the smallest 3 -hr mean plasma velocity for a given day (V0) and the largest 3-hr mean plasma velocity for the following day (V1). Solar wind velocity and plasma density registered by ACE/SWEPAM and SOHO/CELIAS/MTOF experiments were used during the WHI interval as well as during the two adjacent CRs to determining the fast streams. The HSSs obtained are presented in Fig. 1 and Table 1.

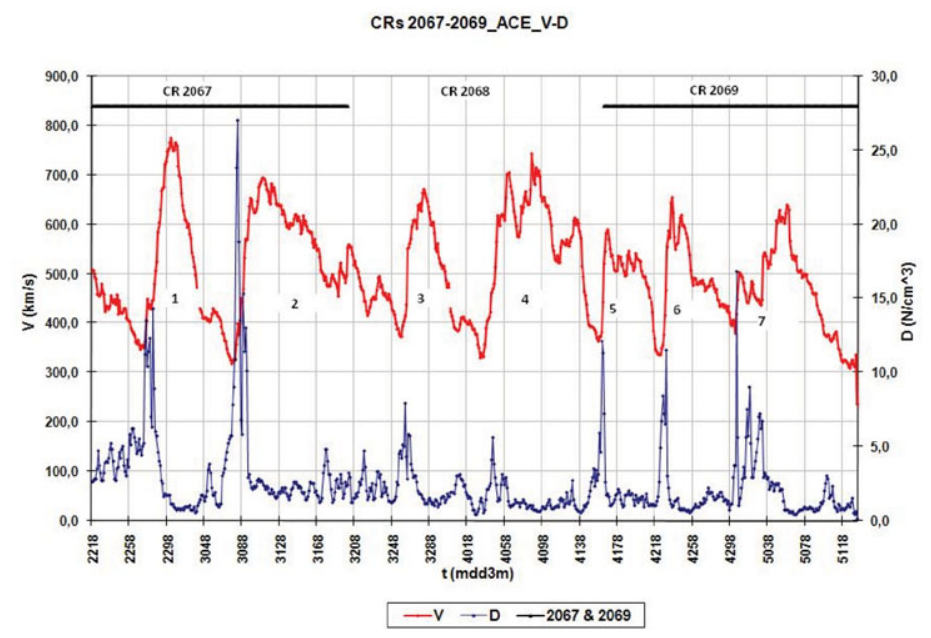

Figure 1. HSSs registered during CRs 2067 - 2069.

Table 1. HSSs during CRs 2067-2069 (HSS numbers correspond to numbers in Fig. 1; A* the Bz polarity alternates during the stream; WHI HSSs in italics).

\begin{tabular}{|c|c|c|c|c|c|c|c|c|c|c|c|c|}
\hline \multicolumn{5}{|c|}{ CR $\mid$ No $|\mathrm{M}| \mathrm{D} \mid 3-\mathrm{h}$} & V0 & V1 & & & & & & $\mathrm{Bz}$ \\
\hline \multirow[t]{2}{*}{2067} & 1 & 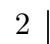 & 27 & 1 & & 5620 & 15 & & $0-4$ & & - & $\mathrm{A}^{*}$ \\
\hline & 2 & 3 & 7 & 8 & & & 7 & & 18.0 & & + & + \\
\hline \multirow[t]{2}{*}{2068} & 3 & 3 & 25 & 8 & & & 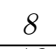 & & & & & - \\
\hline & 4 & 4 & 3 & 3 & .0 & 501.3 & 13 & 73 & 12.9 & .3 & + & + \\
\hline \multirow{3}{*}{2069} & 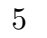 & 4 & 16 & 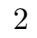 & & & 6 & & & & & + \\
\hline & 6 & 4 & 22 & 5 & & 64 & 10 & & 8. & & - & - \\
\hline & 7 & 4 & 30 & 5 & \begin{tabular}{|l|}
387.3 \\
\end{tabular} & 499.7 & 4 & 637.0 & 9.5 & 249.7 & + & + \\
\hline
\end{tabular}

The significance of the columns in Table 1 are: columns 1 and 2 give the numbers of CRs and streams determined (the same as in Fig.1); columns 3-5 give the start data of the streams, by: M - month; D - day; 3-h - 3-hr interval of the start day; column 6: V0 - minimum (pre-stream) 3-hr velocity of the stream $(\mathrm{km} / \mathrm{s})$; column $7: \mathrm{V} 1$ - maximum 3-hr velocity in the second day of the stream; column 8: dt1 - time interval between V0 and V1 (in number of 3-hr intervals); column 9: Vmax - maximum velocity of the stream; column 10: Dur - duration of the stream, in days; column 11: $\triangle \mathrm{Vmax}=\mathrm{Vmax}$ - V0 is maximum gradient of the plasma velocity; columns 12 and 13: the interplanetary magnetic field (IMF) polarity and the dominant polarity of $\mathrm{Bz}$ component during the stream, respectively. Note that 'dominant polarity' was chosen as the polarity covering more than $80 \%$ of HSS duration (Bz in GSM coordinate system, given by OMNI2 data 
base, http://omniweb.gsfc.nasa.gov). Solar sources of the streams no. 3 and 4 were coronal holes $(\mathrm{CHs})$ observed in the solar corona at low latitudes. The complex structure of the $\mathrm{CH}$, the source of stream no. 4, resulted in the complex structure of the stream. All the streams registered during CRs 2067-2069 were recurrent, as a consequence of their $\mathrm{CH}$ sources (Fig. 2).

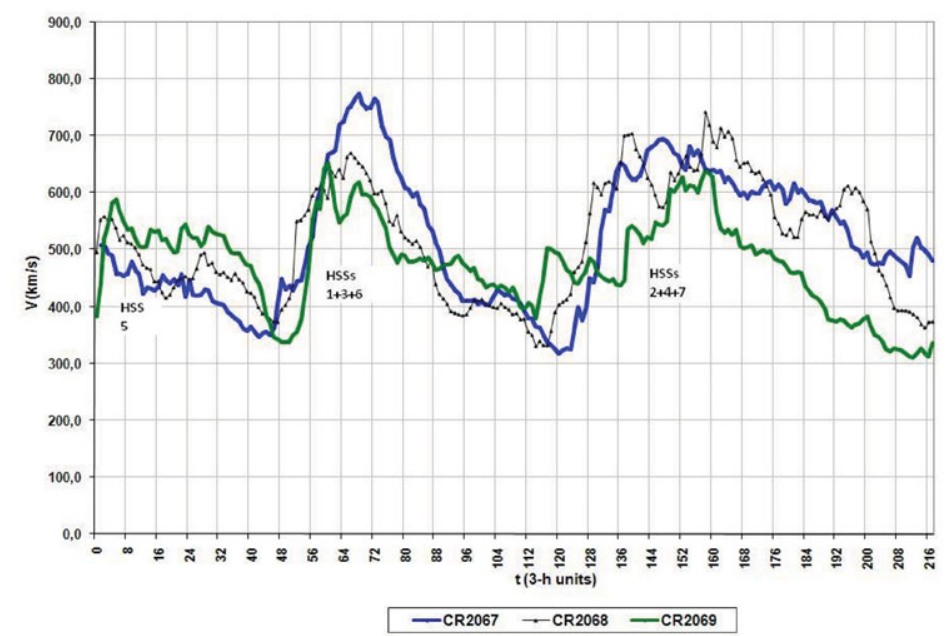

Figure 2. HSSs during CR 2067-2069, for each CR, superimposed over each other. The recurrent nature of the HSSs $(1+3+6)$ and HSSs $(2+4+7)$, respectively, is easily seen. HSS no. 5 (CR 2069) is superimposed over the end of HSS no. 2 (at the beginning of CR 2068).

All the streams had Vmax between $590-770 \mathrm{~km} / \mathrm{s}, \triangle V \max$, between $231-424 \mathrm{~km} / \mathrm{s}$ and, long durations ( $>6$ days). Some of them had complex structure (nos. 2 and 4 ) determined by the successive fast plasma flows generated by the different regions of the $\mathrm{CH}$ sources passing over the central zone of the Sun.

HSSs determined during the WSM interval (1996) are represented in Fig. 3 and all HSS parameters during CRs 1912-1914 are given in Table 2. HSSs nos. 2-5 correspond to the WSM interval (the corresponding numbers are noted in Fig. 3). The complex structures were registered during HSSs nos. 3 and 4, similar to HSSs no. 2 and 4 during WHI (Fig. 1). The streams during CRs 1912-1914 had durations $\leqslant 6.4$ days with only two exceptions (HSSs nos. 6 and 10). Maximum velocities (Vmax) of the four HSSs during WSM were less $619 \mathrm{~km} / \mathrm{s}$ and $\triangle \mathrm{Vmax} \leqslant 228 \mathrm{~km} / \mathrm{s}$ (Table 2$)$.

\section{Geomagnetic Effects of the WHI Streams}

We analyzed the geomagnetic activity during WHI following the classification of the Sunspot Index Data Center (Brussels) as: active periods, with $15<\mathrm{Ap}<30$; minor geomagnetic storm, $29<\mathrm{Ap}<50$; major geomagnetic storm, $49<\mathrm{Ap}<100$; and, severe geomagnetic storm, Ap $\geqslant 100$ (http://sidc.oma.be/educational/classification.php\#geoA). During all HSSs registered in the three CRs, the geomagnetic field presented active periods but only two minor geomagnetic storms appeared as consequences of the HSSs nos. 1 and 3. During HSS no. 3, Bz was southward and favorable to the wind energy transfer to the terrestrial magnetosphere and the correlation coefficient (r) between stream velocity and Dst index was -0.78 . Although HSS no. 4 registered the second highest Vmax and $\triangle V \max$, only sporadically, some energy of the fast stream entered the terrestrial magnetosphere because $\mathrm{Bz}$ was northward (positive) and, $\mathrm{r}$ (V;Dst) $=-0.59$. A good 


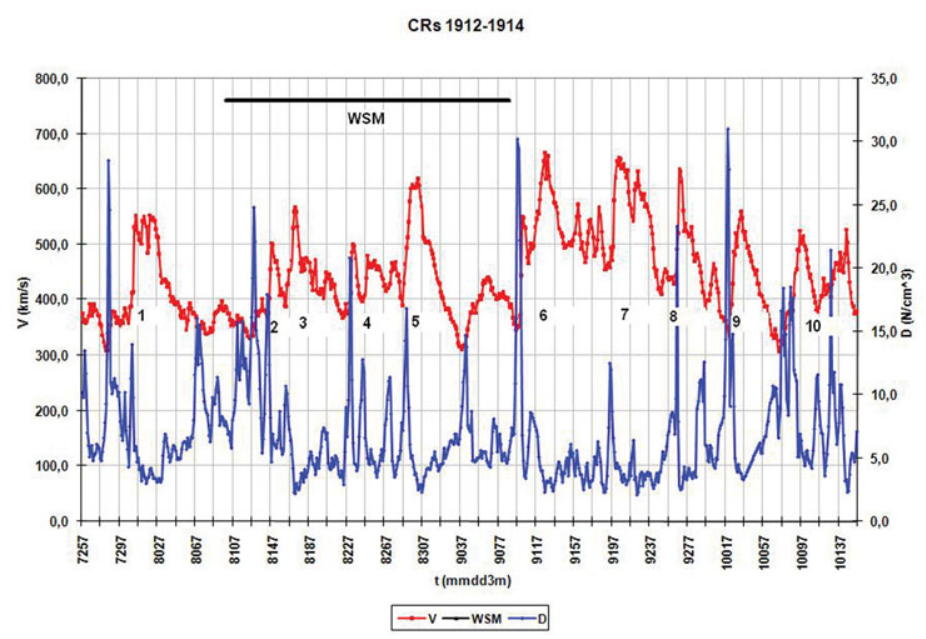

Figure 3. HSSs registered during CRs 1912-1914.

Table 2. HSSs during CRs 1912-1914 (HSS numbers correspond to numbers in Fig. 3; A* the Bz polarity alternates during the stream; WSM HSSs in italics).

\begin{tabular}{|c|c|c|c|c|c|c|c|c|c|c|c|c|}
\hline $\mathrm{CR}$ & No & $\mathrm{M}$ & $D$ & $3-\mathrm{h}$ & V0 & V1 & $\mathrm{dt} 1$ & Vma & Dur & $\triangle \mathrm{Vn}$ & (1) & $\mathrm{Bz}$ \\
\hline \multirow{3}{*}{1912} & 1 & 7 & 30 & 6 & 358.3 & 552.3 & 6 & 553.0 & 6.1 & 194.7 & + & - \\
\hline & 2 & 8 & 13 & 1 & 357.0 & 503.0 & 14 & 503.0 & 3.2 & 146.0 & + & $\mathrm{A}^{*}$ \\
\hline & 3 & 8 & 16 & 3 & \begin{tabular}{|l|}
388.3 \\
\end{tabular} & 566.3 & 8 & \begin{tabular}{|l}
566.3 \\
\end{tabular} & 5.6 & 178.0 & + & $\mathrm{A}^{*}$ \\
\hline \multirow{3}{*}{1913} & 4 & 8 & 22 & 3 & 367.3 & 500.0 & 9 & 500.0 & 6.4 & 132.7 & + & - \\
\hline & 5 & 8 & 28 & 6 & 390.0 & 607.3 & 8 & 618.7 & 4.5 & 228.7 & + & - \\
\hline & 6 & 9 & \begin{tabular}{|l|}
9 \\
\end{tabular} & 6 & \begin{tabular}{|l|}
348.0 \\
\end{tabular} & 551.3 & 5 & \begin{tabular}{|l}
665.7 \\
\end{tabular} & 9.4 & 317.7 & + & - \\
\hline \multirow{4}{*}{1914} & 7 & 9 & 19 & 1 & 454.3 & 656.3 & 12 & 656.3 & 5.4 & 202.0 & + & - \\
\hline & 8 & 9 & 25 & 1 & \begin{tabular}{|l|l|}
411.3 \\
\end{tabular} & 636.7 & 15 & \begin{tabular}{|l|}
636.7 \\
\end{tabular} & 4.5 & 225.4 & + & - \\
\hline & 9 & 10 & 2 & 2 & 336.0 & 520.0 & 6 & 559.0 & 4.8 & 223.0 & - & + \\
\hline & 10 & 10 & \begin{tabular}{|l|}
8 \\
\end{tabular} & 1 & \begin{tabular}{|l|}
337.7 \\
\end{tabular} & 525.3 & 13 & 527.0 & 7.4 & 189.3 & + & - \\
\hline
\end{tabular}

correlation was obtained for HSS no. $6(\mathrm{r}=-0.75)$ and even for HSS no. $1(\mathrm{r}=-0.72)$ where the highest Vmax and $\triangle \mathrm{Vmax}$ disturbed the terrestrial magnetosphere although $\mathrm{Bz}<0$ occurred during $<70 \%$ of the HSS duration.

\section{Acknowledgements}

The authors express their gratitude to the referee for their constructive comments and helpful suggestions. The authors acknowledge the solar wind data obtained by courtesy of ACE/SWEPAM and SOHO/CELIAS/MTOF teams; SOHO is a mission of international cooperation between ESA and NASA. One of the authors (GM) acknowledges the IAU travel grant to IAU General Assembly 2009 in Rio de Janeiro, Brazil.

\section{References}

Lindblad, B. A. \& Lundstedt, H. 1989, Sol. Phys 120, 145-152.

Mavromichalaki, H. \& Vassilaki, A. 1998, Sol. Phys 183, 181-200.

Maris, O. \& Maris, G. 2008, at: http://www.spaceweather.eu/ in Cap. "Data Catalogs for SW". 